Faculty of Arts, University of Ljubljana

Slovenia

luka.repansek@ff.uni-lj.si

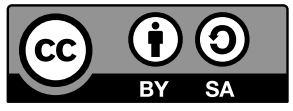

\title{
THE OXFORD HANDBOOK OF ENDANGERED LANGUAGES
}

Rehg, Kenneth L.; Campbell, Lyle (eds.) (2018). The Oxford Handbook of Endangered Languages. New York, NY: Oxford University Press. ISBN 978-01-906-1002-9, 946 pp., £115.

The last few years have seen an increase in handbooks - monographs that should by any standard aim to function as well-rounded state-of-the-art reports on all the various relevant aspects that constitute a particular specialized field of a given scientific discipline - that seem to represent a new generation of reference works, one not necessarily too engaging. The impression some of these recent large-scale collaborative compilations of specialized knowledge convey is more that of the postliminary organization of disparate papers with a highly imbalanced degree of topic particularization into a few overarching chapters, rather than what would and actually should be expected from a comprehensive reference work for a given field. This would be the carefully pre-planned design of principal topics (such as are recognized and / or foreseen as crucial component parts of a particular field), into which reports from specialists are able to aid in the systematization of knowledge within a grander scheme. It cannot be that in the particular case of the handbook reviewed here it is the lack of an established tradition and theoretical identity for the focal discipline that can bear the responsibility for the disorderly and essentially rather unmethodological impression that the monograph makes. More specifically, simply documenting endangered languages for whatever purpose is in its core nothing more and nothing less than a project for descriptive linguistics, while the external circumstances that (unfortunately, although inevitably) lead to the opportunity (in the most neutral possible sense of this word) to do this kind of work, as well as the external conditions that apply, to it are basically the domain of sociolinguistics. I am not too convinced by the plea for a scientifically independent "endangerment linguistics" (see p. 893 and passim), unless the unique combination of inherently interdisciplinary characteristics of working with endangered languages should be recognized as enough of a reason for its foundation and the further fragmentation of contemporary linguistics, already overburdened with not always defensible fractures. 
Be that as it may, leaving aside the lack of overarching systematicity, the handbook does manage to cover most of the relevant topics. Following the introductory chapter by the editors the handbook aims to rationalize the ninety-three papers into five chapters: Endangered Languages, Language Documentation, Language Revitalization, Endangered Languages and Biocultural Diversity, and Looking to the Future. More specifically, the topics dealt with in the individual subdivisions can be subsumed under the following provisional descriptive headings: a) situations that lead to language endangerment (ch. 3); b) ways to assess the level of language endangerment (ch. 2); c) moral, ethical, legislative and social issues connected with language endangerment (qua language rights); d) ethical issues connected with data gathering (ch. 4, 28, 39) and language revitalization (ch. 18); e) why document endangered / disappearing languages (ch. 5, 6); including the notion of reciprocity in the influence of language documentation as phase one of the conservation process on language revitalisation; f) how to document endangered languages (with practical and applicable instructions on how to secure the prerequisites of such projects in general (ch. 7, 35)); g) procuring a reliable grammar of an endangered language (ch. 12); g) dictionary compilation (ch. 13), including the issue of orthographical practices and norms to be applied to the transcriptions (ch. 14); h) the practicalities of how to archive the harvested data to await future systematization (ch. 15); i) the question of the proper contextualization of the linguistic material of a disappearing language, i.e. documenting language use (ch. 16); j) theoretical considerations and practicalities of how to revitalize a disappearing language (ch. 19, 22, 23, 24, 25, 26, 27, some of which include informative case studies); k) pleas for interdisciplinary collaboration, especially in relation to appropriately documenting the finer nuances of environment-specific terminology (plants, animal species, stellar systems, etc.) (ch. 33);1) the search for parallels and potential correlates between the diversity of species and linguistic diversity, as well as the reasons and mechanisms behind the process of extinction, asking the pertinent question as to why conserve linguistic variety as part and parcel of biocultural diversity in the first place (ch. 29, 30,32, with a particular focus on the effects of climate change); and, last but not least, chapter 21, which is in search of the theoretical linguistic framework behind both language loss and language revitalization. The fifty-odd page comprehensive index that concludes the book is of course a highly welcome addition, especially in the light of a noticeable lack of absolutely fixed terminology in the field. The introductory chapter by L. Campbell and K. L. Rehg has to be commended for its coherence and utmost clarity in providing the reader with the bare bones of linguistic and extralinguistic issues connected with endangered and disappearing languages. I would, however, tend to be less compromising in justifying the need to care about disappearing languages. There can be no objection that it is always a good thing to be able to state and defend the objectives of one's research endeavours through a coherently voiced rationale behind a given scientific activity, but it seems that the arguments should never be tailored to suit and convince an external (re)viewer for fear of conveying a lartpourlartistic modus operandi. On the 
contrary, the argument that both the scientific and cultural dimensions of language simply must be encompassed in their entirety in order to be systematized and understood should suffice. There is nothing that we as humans do not investigate (at least primarily) out of sheer curiosity. Then, of course, in the case of endangered languages the cause for increased engagement in linguistic work has in its favour the additional argument of the blatant violation of human rights that takes place whenever a socially-induced extralinguistic factor endangers the very existence of any given linguistic system.

The concluding paper from David Crystal, simply entitled "Afterword" (pp. 885894), is in all actuality a highly focused, wide-ranging and ambitious review of the preceding chapters, to which very little can indeed be added. Perhaps what should be pointed out is that the term "language", whenever one speaks of language endangerment, language disappearance and so on, must by logical necessity refer to any linguistic system, i.e., including on the level of the individual dialects. In this particular case the thin line between fieldwork and language documentation of endangered as opposed to generally poorly documented systems is especially evident. It is true that these may not fulfil the standard criteria for language endangerment, but are in fact equally endangered in the sense that they achieve no or hardly any visibility and are for the purposes of linguistics practically non-existent. When language documentation is seen as having the sole purpose of harvesting what often prove to be crucial pieces of the otherwise fragmentary linguistic impression of a given language (see also the significance of fieldwork on poorly documented language systems for, say, a deeper understanding of the historical grammar of a language) - and that in fact repeatedly tends to become the only viable objection in the face of the increasingly unfavourable external (for now still mostly socially related) circumstances - all linguistic systems matter equally and all should of course be aptly documented. Because it is true that given the (now hopefully slowly decreasing) unpopularity of fieldwork among contemporary linguists we lack the people and hence the time to reverse the trend of thinning data, precedence should naturally be given to those languages that face imminent extinction, but since one will usually lose something momentarily left out of sight while trying to rescue another thing this should be happening on a much larger scale, calling for the complete reorganization of synchronic linguistics as a field. Unfortunately, however, it seems we are just as eager to act collectively in the case of language disappearance caused by globalization and increasing linguistic superstratization as we are in the case of the rapid decrease in the diversity of species brought about by the equally disastrous consequences of the global deterioration of the natural environment. 